\title{
Synthesis and Exciton Dynamics of Donor-Orthogonal Acceptor Conjugated Polymers: Reducing the Singlet-Triplet Energy Gap
}

\author{
David M. E. Freeman ${ }^{1 *}$, Andrew J. Musser ${ }^{2 \dagger+}$, Jarvist M. Frost 3 , Hannah L. Stern ${ }^{2}$, Alexander K. \\ Forster $^{1}$, Kealan J. Fallon ${ }^{1}$, Alexandros G. Rapidis ${ }^{4}$, Franco Cacialli', Iain McCulloch5, Tracey M. \\ Clarke$^{1}$, Richard H. Friend ${ }^{2 *}$, Hugo Bronstein ${ }^{1 *}$ \\ 1 Department of Chemistry, University College London, 20 Gordon St., London, WC1H oAJ, UK \\ 2 Cavendish Laboratory, University of Cambridge, JJ Thomson Avenue, Cambridge, CB3 oHE, UK \\ 3 Department of Materials, Imperial College London, Exhibition Road, London, $\mathrm{SW}_{7} 2 \mathrm{AZ}$, UK \\ 4.Department of Physics and Astronomy and London Centre for Nanotechnology, University College London, Gower Street, \\ London WC1E 6BT, UK \\ 5 SPERC, King Abdullah University of Science and Technology, Thuwal 23955-69oo, Saudi Arabia
}

\begin{abstract}
The presence of energetically low-lying triplet states is a hallmark of organic semiconductors. While they present a wealth of interesting photophysical properties, these optically dark states significantly limit optoelectronic device performance. Recent advances in emissive charge-transfer molecules have pioneered routes to reduce the energy gap between triplets and 'bright' singlets, allowing thermal population exchange between them and eliminating a significant loss channel in devices. In conjugated polymers this gap has proved resistant to modification. Here, we introduce a general approach to reduce the singlet-triplet energy gap in fully conjugated polymers, using a donor-orthogonal-acceptor motif to spatially separate electron and hole wavefunctions. This new generation of conjugated polymers allows for a greatly reduced exchange energy, enhancing triplet formation and enabling thermally-activated delayed fluorescence. We find that the mechanisms of both processes are driven by excited-state mixing between $\pi-\pi^{*}$ and charge-transfer states, affording new insight into reverse intersystem crossing.
\end{abstract}

\section{Introduction}

The low-energy triplet excited state in conjugated polymers poses a substantial barrier to next-generation optoelectronic device applications. One advantage of conjugated polymer semiconductors is their strong absorption and emission, due to the almost total overlap of $\pi$ and $\pi^{*}$ and orbitals. However, the localised and overlapping wavefunctions result in a large triplet-stabilising exchange energy $\Delta \mathrm{E}_{\text {ST }}$ of $\approx 0.7 \mathrm{eV} .^{1-3}$ The low energy of the non-emissive triplet state means it forms a trap and loss mechanism in virtually all organic optoelectronic devices (OPV, OLED, OLET, OLASER)..$^{4-6}$ This could be avoided were it possible to reduce $\Delta \mathrm{E}_{\text {ST }}$ through material design. For application to OLEDs, triplet excitons could be thermally converted back into singlets via reverse intersystem crossing (RISC), amply demonstrated in small-molecule systems with small $\Delta \mathrm{E}_{\mathrm{ST}}{ }^{7}$ In an organic solar cell architecture, substantially reducing $\Delta \mathrm{E}_{\mathrm{ST}}$ would mean that the stochastic generation of triplets from recombining charges is no longer a loss pathway. ${ }^{7-8}$

Such energy-level manipulation was assumed impossible in conjugated polymers, due to a consistently observed $\Delta \mathrm{E}_{\text {ST }}$ of $\approx 0.7 \mathrm{eV}$. Recent work has shown that a reduction in $\Delta \mathrm{E}_{\mathrm{ST}}$ can be achieved in non-conjugated polymers. Ni- kolaenko et al. reduced $\Delta \mathrm{E}_{\text {ST }}$ by linking small molecules capable of thermally activated delayed fluorescence (TADF) into a non-conjugated polymer host. 9 Luo et al. demonstrated a similar concept with pendant electron-deficient moieties on a non-conjugated 3,6-polycarbazole backbone. ${ }^{10}$ More recently, Adachi et al. exploited the small $\Delta \mathrm{E}_{\text {ST }}$ of benzophenone to demonstrate TADF in a keto-polymer."

Herein we present a novel concept in polymer design allowing the construction of fully conjugated polymers with significantly reduced $\Delta \mathrm{E}_{\text {ST }}$ (Figure 1 ). The principles are applicable to the entire library of existing polymers and we anticipate these materials will open a new design space in conjugated polymer synthesis. Conjugated polymers are often synthesised with linear donor-acceptor combinations to tune the optical energy gap. By removing the acceptor from the main chain, and placing it physically orthogonal to the donor backbone, we achieve a massive reduction in the spatial overlap of the frontier molecular orbitals, whilst maintaining conjugation along the polymer backbone. We therefore retain all the benefits of conjugated polymers, such as processability and charge transport along the polymer backbone, while the resulting materials also benefit from a greatly reduced $\Delta \mathrm{E}_{\mathrm{ST}}$. 

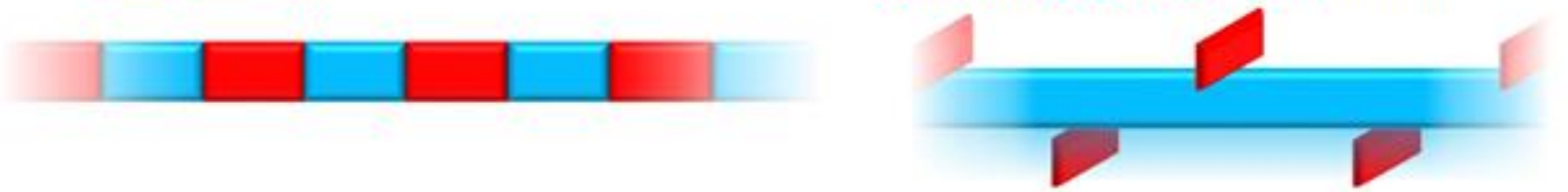

$S_{1}$

$$
\Delta E_{S T}=0.7 \mathrm{eV}
$$

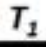

$S_{0}$

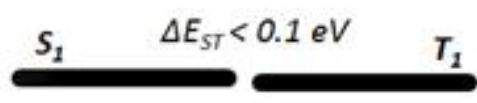

$S_{0}$

Figure 1. Comparison of conventional donor-acceptor (D-A) conjugated polymer and donor-orthogonal acceptor (D-oA) conjugated polymer and its effect on the singlet-triplet energy gap.

We demonstrate this concept by modifying two of the most common conjugated polymers into donor-orthogonal acceptor (D-oA) materials. Our spectroscopic and computational investigation reveals the interplay of $\pi-\pi^{*}$ and charge-transfer singlet and triplet states that drives triplet formation and RISC. We synthesized the series of conjugated polymers based on established polyfluorene-containing materials shown in Figure 2 (Supporting Information $\mathrm{S}_{2}-\mathrm{S}_{3}$ ). The model system $\mathbf{S F H}$ is a simple PFO conjugated backbone with alternating 9,9'-spirobifluorene moieties, similar to a previously reported structure. ${ }^{12}$ In order to create the novel D-oA polymer SFCN, electron withdrawing cyano groups were introduced onto the orthogonal spirobifluorene unit, a strategy that has been employed for achieving TADF in small molecules. ${ }^{13-15}$ Our other model conjugated polymer incorporating a more electronrich backbone co-monomer was ASFH, similar to the wellknown TFB. ${ }^{16}$ Again, this structure was modified by the introduction of cyano groups on the orthogonal spirobifluorene, yielding the novel D-oA polymer ASFCN.

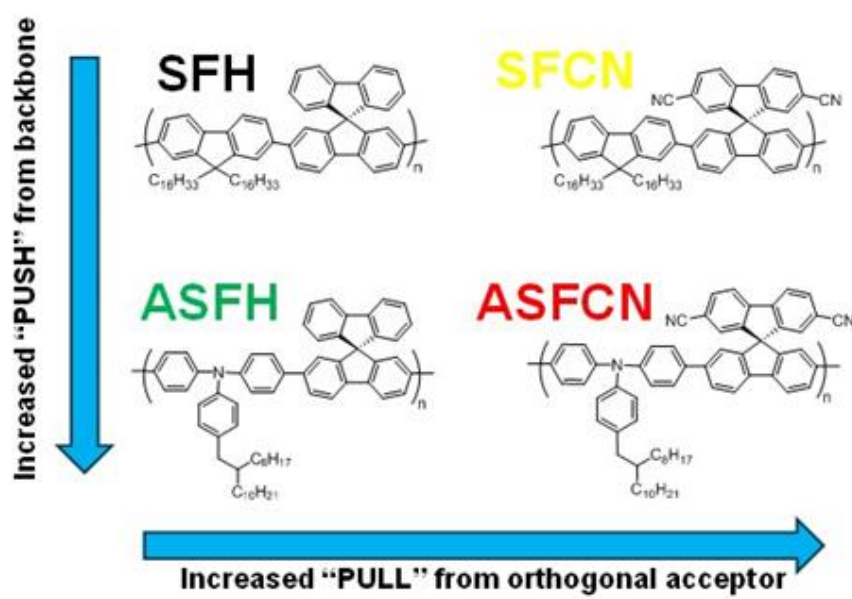

Figure 2. Structures of synthesized conjugated polymers

\begin{tabular}{|c|c|c|c|c|c|c|}
\hline $\begin{array}{l}\text { Poly- } \\
\text { mer }\end{array}$ & $\begin{array}{l}\mathrm{M}_{\mathrm{n}}{ }^{\mathrm{a}} / \\
\mathrm{kDa}\end{array}$ & $\begin{array}{l}\mathbf{M}_{w^{a}}{ }^{a} / \\
\mathbf{k D a}\end{array}$ & PDI $^{a}$ & $\begin{array}{l}\text { HO- } \\
\mathrm{MO}^{\mathrm{b}} \\
/ \mathrm{eV}\end{array}$ & $\begin{array}{l}E_{\text {gap }}{ }^{c} \\
/ \mathbf{e V}\end{array}$ & $\begin{array}{l}\boldsymbol{\varepsilon} \\
/ \mathbf{M}^{-} \\
{ }^{\mathrm{I}} \mathbf{c m}^{-1}\end{array}$ \\
\hline SFH & 16 & 23 & 1.44 & -5.77 & 2.97 & 16104 \\
\hline SFCN & 20 & 29 & 1.45 & -5.81 & 2.97 & 18384 \\
\hline ASFH & 18 & 31 & 1.72 & -5.37 & 2.89 & 11844 \\
\hline ASFCN & 23 & 49 & 2.13 & -5.42 & 2.79 & 7841 \\
\hline
\end{tabular}

Table 1. Physical properties of conjugated polymers (a) Determined by SEC (PS) using $\mathrm{PhCl}$ as eluent. (b) Determined by PESA. (c) Determined from thin film UV-Vis absorption onset. (d) Determined in $\mathrm{CHCl}_{3}$ solution.

Thin-film absorption spectra (Fig. 3) reveal slight effects of our D-oA approach on the nature of the absorbing state. In SFH and SFCN, the primary absorption band closely matches the parent polymer PFO, with a distinct enhancement of absorption strength in the D-oA material SFCN versus SFH. The aryl-amine-containing polymers exhibit narrower band-gaps, and the spectrum of ASFH closely follows that of isoelectronic TFB. Interestingly, the D-oA effects are more pronounced when combined with a 'push' backbone in ASFCN: a clear red-shift of $\sim 100 \mathrm{meV}$ and reduction in extinction coefficient. These effects imply the absorption has taken on some charge-transfer (CT) character, as is commonly seen in linear donor-acceptor conjugated polymers.and especially ASFCN where the photoluminescence is red-shifted, broad and featureless. SFH and ASFH possessed ionisation potentials in agreement with their counterparts PFO and TFB. Introduction of the orthogonal acceptor groups resulted in a stabilisation of the $\mathrm{HOMO}$ by $\sim 0.05 \mathrm{eV}$ relative to their respective unsubstituted polymer. 


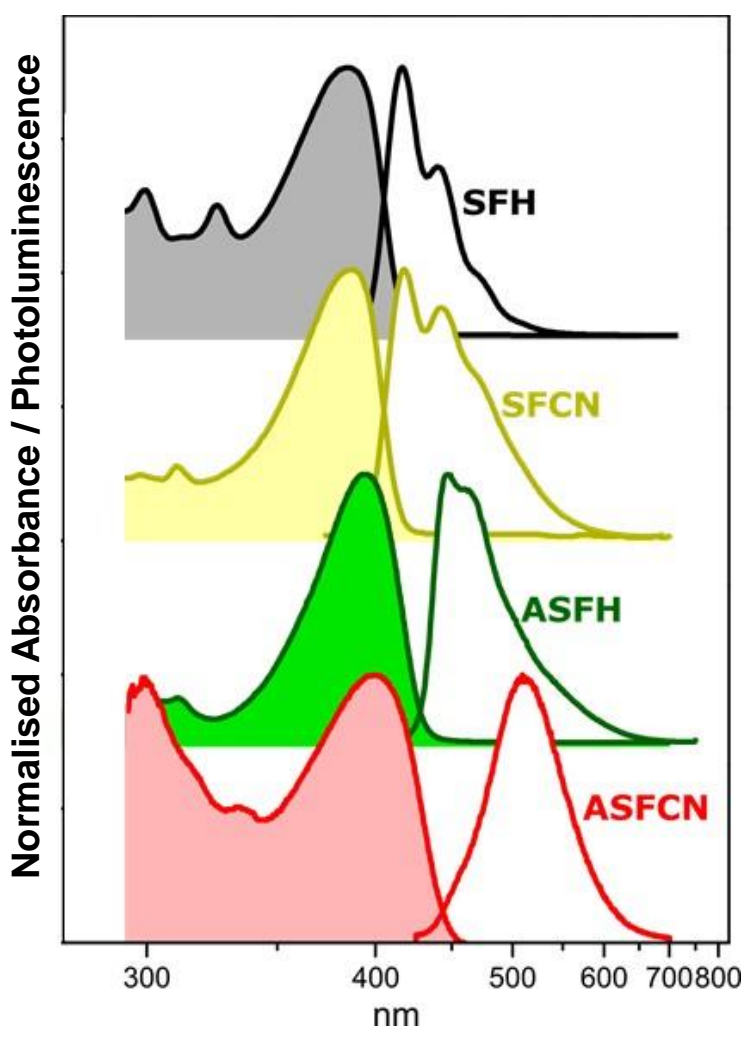

Figure 3. UV-vis absorption (shaded) and photoluminescence (unshaded) of polymer thin films. Fluorene-backbone polymers SFH and SFCN exhibit the same band gap in absorption $\sim 417 \mathrm{~nm}$. The electron-rich backbone of ASFH results in a red-shift of $80 \mathrm{meV}$ to $429 \mathrm{~nm}$, while incorporation of the polar acceptor units in ASFCN red-shifts the absorption by a further $100 \mathrm{meV}$ to $444 \mathrm{~nm}$. The effect of $-\mathrm{CN}$ substitution is markedly more pronounced in the excited state, with contributions from charge-transfer emission in SFCN and especially ASFCN where the photoluminescence is red-shifted, broad and featureless.

\section{Emissive Properties}

The effects of D-oA substitution are particularly prominent on the polymer photoluminescence (Fig. 3). SFH and ASFH exhibit predominantly conventional ${ }^{1}\left(\pi-\pi^{*}\right)$ emission, with a small Stokes shift and well-defined vibronic progression. Incorporation of cyano groups induces a slight red-shift and broadening in SFCN, consistent with a new lower energy ${ }^{1} \mathrm{CT}$ state. ${ }^{17-21}$ This effect is amplified in ASFCN, in which the substantial red-shift and absence of vibronic features indicate that the emission is $\mathrm{CT}$ in nature. In Fig. 4 we illustrate how these spectra evolve in time, measured on an instrument with a $\sim 5$ ns response. 'Pullonly' SFCN exhibits two distinct emissive species, similar to 'push-only' ASFH (Fig. S2): initial vibronically structured ${ }^{1}\left(\pi-\pi^{*}\right)$ emission decays on an instrument-limited timescale to yield a weak, red-shifted defect or ${ }^{1} \mathrm{CT}$ state (peak at $500 \mathrm{~nm}$ ). The latter can be relatively long-lived, with trace emission detectable up to $\sim 100$ ns (Fig. 4c). Given that the CT state is rapidly populated in SFCN (see below) yet exhibits very little emission, we conclude that the ${ }^{1} \mathrm{CT}$ state mixes poorly with the ${ }^{1}\left(\pi-\pi^{*}\right)$ and so has negligible transition dipole moment. ASFCN exhibits no such evolution of spectral shape on these timescales, but instead a slight, continuous red-shift with no evidence of a distinct ${ }^{1}\left(\pi-\pi^{*}\right)$ (see Fig. S2). The rise in PL intensity evident from 1-3 ns to 5-10 ns is convoluted with the instrument response but nonetheless reveals that the primary emissive state is not directly photoexcited, i.e. the emissive ${ }^{1} \mathrm{CT}$ is not the state that dominates the absorption spectrum but is instead formed though an internal conversion process. It is significant that the long-lived state is much more strongly emissive in this polymer. Fluorescence is readily detected for 10o's of ns, when triplets are the dominant species (see below). The PL lifetime enhancement for ASFCN is a signature of delayed fluorescence, which typically arises from either triplet-triplet annihilation ${ }^{22}$ or thermally activated RISC (akin to that proposed for small molecules7). To differentiate between the two mechanisms, we measured the PL decay dynamics at lower excitation density and performed temperature-dependent steady-state measurements.
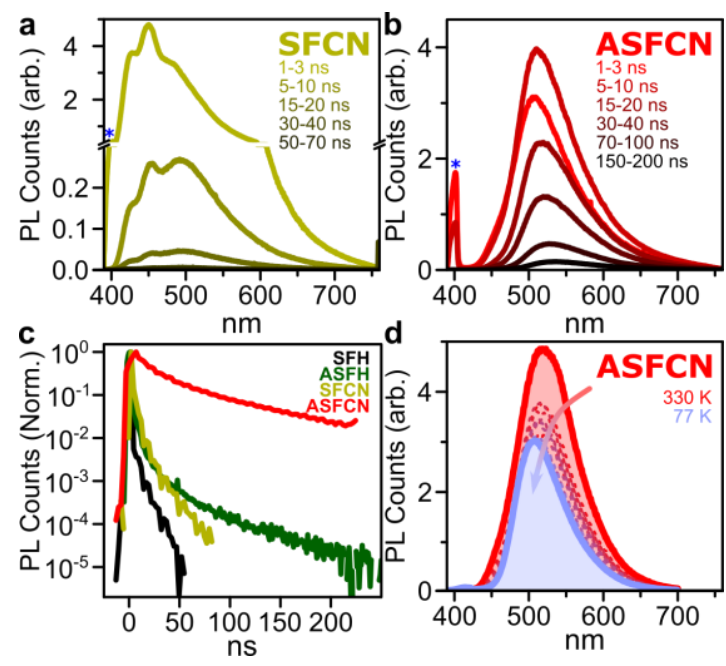

Figure 4. Photoluminescence dynamics in $\Delta \mathrm{E}_{S T}$-tuned polymer films. (a) Time-resolved emission spectra of SFCN film in vacuum, following excitation at $400 \mathrm{~nm}$, with a temporal resolution of $\sim 5 \mathrm{~ns}$. Emission is dominated by singlet ( $\pi$ $\pi^{*}$ ) emission. Vertical axis is broken to highlight very weak contribution from CT singlet emission at $500 \mathrm{~nm}$ evident on longer timescales. Similar behaviour is observed in the "pushonly' polymer ASFH (Fig. S2). (b) Equivalent measurements of ASFCN films. We detect only broad, structureless CT emission, with no equivalent to the initial vibronically structured $\pi-\pi^{*}$ emission observed in other polymers. The rise in PL intensity between the first and second spectra in ASFCN reveals delayed formation of the emissive CT state, though it is convoluted with the instrument response. Pump scatter in both spectral plots is indicated by *. (c) PL decay kinetics measured for all polymer films, taken at $450 \mathrm{~nm}$ (SFH and SFCN) and $500 \mathrm{~nm}$ (ASFH and ASFCN). Incorporation of cyanobiflourene units and introduction of CT states increases the PL lifetime (light green vs. black, red vs. dark green). In ASFCN, significant PL is detected on the 10o's of ns timescale, when triplets are the dominant species. (d) Steady-state PL spectra of ASFCN, from $330 \mathrm{~K}$ (red) to $77 \mathrm{~K}$ (blue). The PL intensity gradually decreases upon cooling, consistent with a major contribution to the PL from TADF. Normalised spectra and equivalent measurement of ASFH in Fig. S3. 
The kinetics shown in Figure $4 \mathrm{c}$ were obtained on a $1 \mathrm{kHz}$ laser system using amplified pulses. Using an unamplified 2.5 $\mathrm{MHz}$ diode laser with dramatically lower pulse energies, we obtain the same delayed PL decay kinetics in ASFCN (Fig. S4), demonstrating that the long lifetime is insensitive to excitation density and should not arise from triplet-triplet annihilation. As shown in Figure 4d, temperature reduction both decreases the ASFCN thin-film PL yield and blue-shifts the spectral intensity towards the peak at $508 \mathrm{~nm}$ (Fig. S3). These effects are consistent with a changing balance of emissive species, with the lower-energy emitter populated via thermal activation. By contrast, ASFH exhibits negligible shifts and an increase in PL yield upon cooling, a typical consequence of suppressing of nonradiative decay channels (Fig. $\mathrm{S}_{3}$ ). Our observation of the opposite behaviour in ASFCN is direct proof of TADF in a conjugated polymer, enabled by reducing $\Delta \mathrm{E}_{S \mathrm{~T}}$ through our D-oA motif. These observations also enable us to rationalise the continuous time-resolved PL red-shift in ASFCN. The thermally activated process will be most efficient at sites with the lowest $\Delta \mathrm{E}_{\mathrm{ST}}$ and thus lowest-energy emissive CT states, resulting in selective enhancement of the PL lifetime at the red spectral edge. It is interesting to note that, in contrast to standard small-molecule TADF emitters, ASFCN exhibits no clear delineation between 'prompt' and 'delayed' emission. ${ }^{6,16,19}$ Moreover, comparison with transient absorption data below reveals a strong correlation between substantial $3(\pi-\pi)^{*}$ character in the ASFCN excited state and the emission on all timescales, suggesting a more complex TADF mechanism than is typically proposed. Photoluminescence quantum yields are discussed in the supplementary information page S3o.

\section{CT-mediated Triplet Formation}

To understand the photophysical effects of $-\mathrm{CN}$ substitution and the delayed fluorescence mechanism, transient absorption (TA) spectroscopy was performed. TA is sensitive to the absorption of excited states therefore wellsuited to the study of RISC, where the states of primary interest are optically 'dark'. In Figure 5a we present the TA evolution of SFCN in solution. Immediately following photoexcitation, we detect a photo-induced absorption (PIA) band at $730 \mathrm{~nm}$ which can be assigned to the ${ }^{1}\left(\pi-\pi^{*}\right)$ (black) from comparison with the unsubstituted SFH (Fig. S5). Over $\sim 20$ ps this state decays to yield a weaker PIA band at $530 \mathrm{~nm}$ (dark yellow); a similar state appears in ASFCN (Fig. 5b) but not in either reference polymer, indicating it is a direct consequence of the cyano groups. The decay of this $530 \mathrm{~nm}$ feature in the SFCN data closely tracks the redshifted PL detected in solution (Fig. 5a, open circles), and it can thus be assigned to the SFCN CT state. ${ }^{17,23}$ We attribute the fast formation observed here to the strong electronwithdrawing character of the cyano groups. On the 1o's of ns timescale, the spectrum further evolves into a sharp PIA at $760 \mathrm{~nm}$ (red). This final state is remarkably long-lived ( $\tau$ $>800 \mu \mathrm{s}$ ), with a lifetime that is highly sensitive to oxygen (blue dashed kinetic; full data in Fig. S6). These characteristics identify the band at $760 \mathrm{~nm}$ as the $3\left(\pi-\pi^{*}\right)$ state, in agreement with previous studies on similar fluorene-based polymers. ${ }^{24}$ The very large signal suggests a high intersystem crossing yield, a likely consequence of reducing $\Delta \mathrm{E}_{S T}$ through our D-oA concept, though in SFCN this effect is not sufficient to enable RISC. The data in Fig5a thus establish an excited-state pathway in SFCN of ${ }^{1}\left(\pi-\pi^{*}\right) \rightarrow \mathrm{CT} \rightarrow$ $3\left(\pi-\pi^{*}\right)$, in which both conversions are driven by the unique properties of the D-oA structure.

We observe a similar progression of states in ASFCN in solution (Fig. 5 b): fast ${ }^{1}\left(\pi-\pi^{*}\right)$ decay (black, $\tau \sim 300 \mathrm{fs}$ ) yields an emissive CT state (dark yellow) with PIA below $550 \mathrm{~nm}$. Following CT decay, a long-lived $(\tau>3 \mu$ s) state with PIA similar to $3\left(\pi-\pi^{*}\right)$ in SFCN is evident (red), which again exhibits strong sensitivity to oxygen (blue dashed kinetic). This can be assigned to the $3\left(\pi-\pi^{*}\right)$ state of ASFCN. However, the distinction between the final states is less sharp: no $3\left(\pi-\pi^{*}\right)$ PIA growth is detected during CT decay, and indeed some $3\left(\pi-\pi^{*}\right)$ features can be detected throughout the CT lifetime and vice versa. We consider that the smaller $\Delta \mathrm{E}_{S \mathrm{~T}}$ here results in some dynamic equilibrium or mixing between $3\left(\pi-\pi^{*}\right)$ and emissive CT states, analogous to signatures of singlet-mixed triplet-pair states observed in certain small molecule systems. ${ }^{25-28}$ Nonetheless, on the longest timescales eventual conformational relaxation in the $3\left(\pi-\pi^{*}\right)$ makes back-transfer to CT thermally inaccessible and emission is quenched. Similar results are obtained in the solid state, with only slight shifts in the characteristic PIA bands of each excited state. In SFCN (Fig. 5 C), ${ }^{1}\left(\pi-\pi^{*}\right)$ $\rightarrow$ CT conversion is faster than in solution $(\tau \sim 40 \mathrm{Os})$, but a substantial population of singlet $\left(\pi-\pi^{*}\right)$ states appear unable to form CT and decay with complicated non-exponential kinetics. This branching of ${ }^{1}\left(\pi-\pi^{*}\right)$ decay pathways may reflect the presence of lower-energy ${ }^{1}\left(\pi-\pi^{*}\right)$ 'trap' sites arising from interchain interactions. From the much lower CT state signal in SFCN film versus solution, we expect that the 400 fs time constant is dominated by the formation of these low-energy ${ }^{1}\left(\pi-\pi^{*}\right)$ states rather than CT states. Primary CT state decay is markedly faster $(\tau \sim 90 \mathrm{ps})$, though the relatively small $3\left(\pi-\pi^{*}\right)$ signal suggests this is not due to enhanced intersystem crossing. As previously, the PL measured on long timescales (open circles, magnified for clarity) is consistent with the (trace) CT state population, rather than triplets, confirming that the reduction of $\Delta \mathrm{E}_{\mathrm{ST}}$ in SFCN is insufficient to enable TADF. By contrast, ASFCN films (Fig. 5d) show stronger evidence of CT-3 $(\pi-$ $\pi^{*}$ ) mixing than in solution, with features of both states evident over the full lifetime following prompt ${ }^{1}\left(\pi-\pi^{*}\right)$ decay $(\tau \sim 300 \mathrm{fs})$. Initially the population balance is primarily CT (PIA $\sim 550 \mathrm{~nm}$ ), while beyond 1 ns the $3\left(\pi-\pi^{*}\right)$ character (PIA $\sim 750 \mathrm{~nm}$ ) dominates. Importantly, integrated PL kinetics (open circles) precisely match the triplet $\left(\pi-\pi^{*}\right)$ PIA, confirming the origin of the red-shifted emission as RISC. The activation of RISC only in the solid state suggests an important role for conformational restriction: the $3\left(\pi-\pi^{*}\right)$ state is unable to relax energetically to the same degree as in solution, maintaining the small CT-3 $\left(\pi-\pi^{*}\right)$ energetic separation and enabling RISC. 

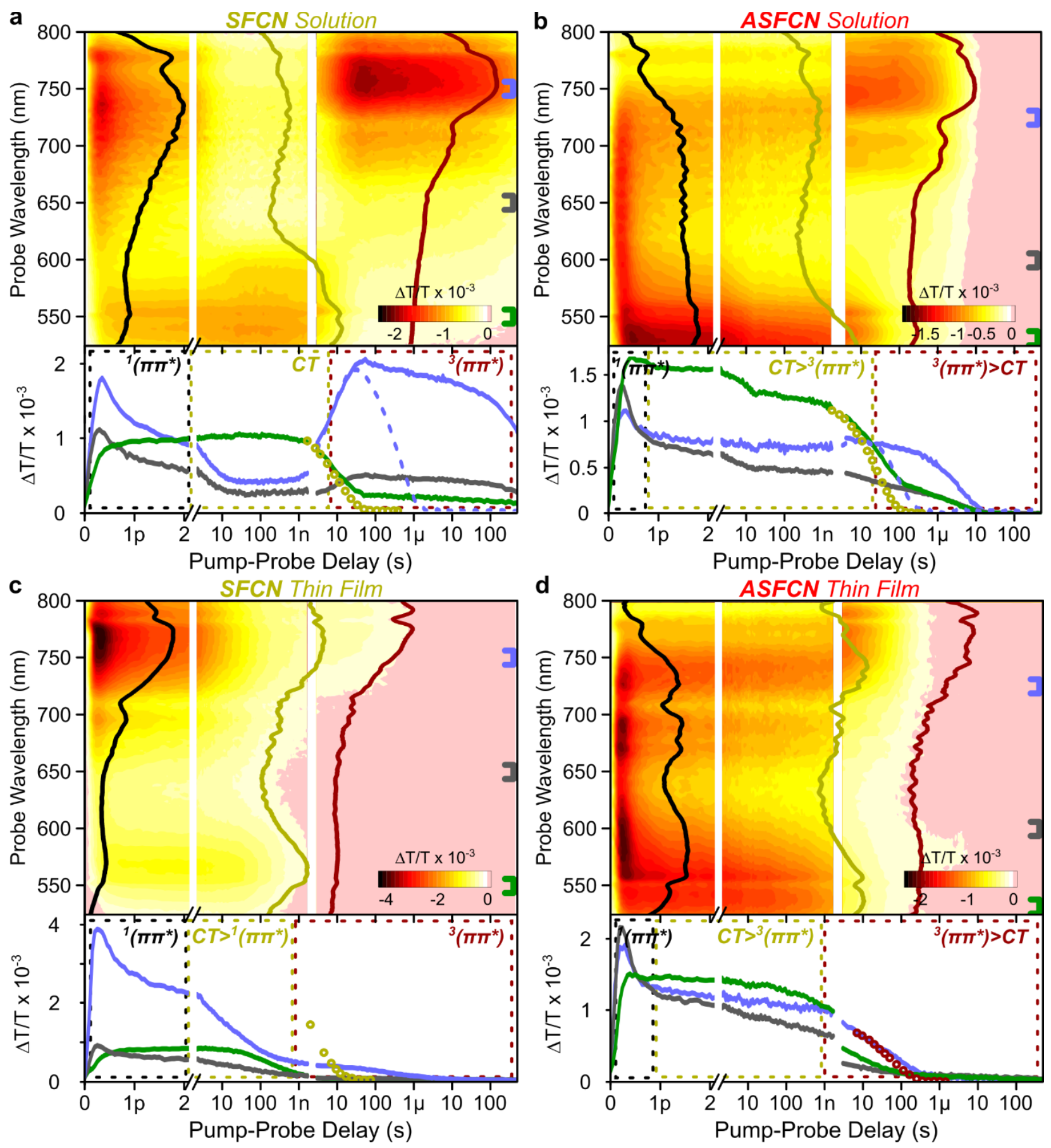

Figure 5. Triplet formation in D-oA polymers. TA spectral maps of (a,c) SFCN and (b,d) ASFCN in (a,b) chloroform solution and (c,d) spin-cast film under vacuum. Spectral cuts (lines) highlight characteristic excited-state features. Kinetics below are integrated over bracketed regions, with boxes denoting dominant state in that temporal regime. (a) SFCN solution, with clean conversion from ${ }^{1}\left(\pi-\pi^{*}\right)$ (black, $500 \mathrm{fs}$ ) to CT (dark yellow, $250 \mathrm{ps}$ ) to $3\left(\pi-\pi^{*}\right)$ (red, $500 \mathrm{~ns}$ ). Triplet (lifetime $>800 \mu$ s) is quenched by oxygen (dashed kinetic). (b) Similar features identified for ASFCN solution CT (dark yellow, 10o ps) and $3\left(\pi\right.$ - $\left.\pi^{*}\right)$ (red, 150 ns) states, with a broader and shorter-lived ${ }^{1}\left(\pi-\pi^{*}\right)$ (black, $\left.300 \mathrm{fs}\right)$. CT and $3\left(\pi-\pi^{*}\right)$ are less distinct, with signatures of each evident throughout the lifetime of the other. Triplet (lifetime $3.7 \mu \mathrm{s}$ ) is quenched by oxygen (dashed kinetic). (c) SFCN thin film exhibits same ${ }^{1}\left(\pi-\pi^{*}\right)$ (black, $300 \mathrm{fs}$ ), CT (dark yellow, $100 \mathrm{ps}$ ) and $3\left(\pi-\pi^{*}\right)$ (red, $5 \mathrm{~ns}$ ) characteristics. The ${ }^{1}\left(\pi-\pi^{*}\right) \rightarrow$ CT conversion efficiency and triplet lifetime are sharply reduced. (d) ASFCN thin films show stronger signs of excited-state mixing. Following prompt ${ }^{1}\left(\pi-\pi^{*}\right)($ black, $300 \mathrm{fs}$ ) decay, the population shifts from primarily CT (dark yellow, $1 \mathrm{~ns})$ to primarily $3\left(\pi-\pi^{*}\right)$ (red, $\left.150 \mathrm{~ns}\right)$. Open circles: integrated PL kinetics from Figure 4, tracking CT in solution (a,b) and SFCN film (c), and $3\left(\pi-\pi^{*}\right)$ in ASFCN film (d). Break in data $1.5-4$ ns reflects transition from sub-ps-TA (100 fs pump, $400 \mathrm{~nm}$ ) to ns-TA (1-ns pump, $355 \mathrm{~nm}$ ), details in Supporting Information. 


\section{Mechanism of $\Delta \mathrm{E}_{S \mathrm{ST}}$ Manipulation}

In small molecules, manipulation of $\Delta \mathrm{E}_{\mathrm{ST}}$ to enable RISC is well known through the study of TADF. The crucial design rule is the reduction of spatial overlap between occupied and unoccupied molecular orbitals. To investigate how this rule translates to polymeric systems, we have performed hybrid density functional theory (hybrid DFT, B3LYP functional) calculations on symmetric oligomers of our spiro units flanked with hydrogen-capped monomers, followed by linear response time-dependent density-functional-theory (TDDFT) to probe the excited states. The small system was chosen for computational efficiency and the ability to develop a single-electron picture of excitations, rather than for quantitative agreement with experiment. The excited states were well represented by mixes of the ground-state occupied and virtual Kohn-Sham orbitals, so we begin by discussing these electron localisations.

The major light-absorbing transition in $\pi$-conjugated materials is between delocalised $\pi$ orbitals and $\pi^{*}$ excited states. In typical systems such as SFH/ASFH, these orbitals are located primarily along the conjugated backbone, with significant spatial overlap and consequently large oscillator strength in the singlet configuration (Fig. 6a). In our DoA polymers SFCN and ASFCN, we find virtually identical extension of the $\pi$ orbital (HOMO) along the polymer chain, due to relatively weak (albeit non-zero) conjugation across the spiro linkage. ${ }^{29}$ The effect of the D-oA motif in the ground-state geometry is to stabilise the orbitals on the spiro unit below the $\pi^{*}$ orbitals along the backbone. Thus, the first unoccupied state (LUMO) of SFCN and ASFCN is localised on the orthogonal acceptor group, eliminating spatial overlap between HOMO and LUMO. Crucially, spiro-conjugation allows for some electronic communication across the orthogonal moieties. ${ }^{29}$ This transition has very low oscillator strength but affords negligible energetic separation between $\mathrm{S}$ and $\mathrm{T}$ excited state energies.

The Jablonski diagram of excited states as calculated by TD-DFT (Figure 6b) agree with our orbital analysis. TDDFT is an approximate theory. In particular it has a known difficulty in predicting the relative energy of Singlet and Triplet states (as the exchange and correlation potential which leads to this splitting is an uncontrolled approximation)..$^{30}$ The choice of a TDDFT method is necessitated by the large size of a minimal model. ${ }^{31}$ Some of the weakness of TD-DFT can be sidestepped by applying a further Tamm-Dancoff approximation (TDA). Thus, the calculations were carried using LR-TD-DFT at B3LYP/6-31g* (Figure 6) and 6-311g* both with and without TDA (see supporting information S6). All calculations qualitatively gave similar orbital energetic orderings.
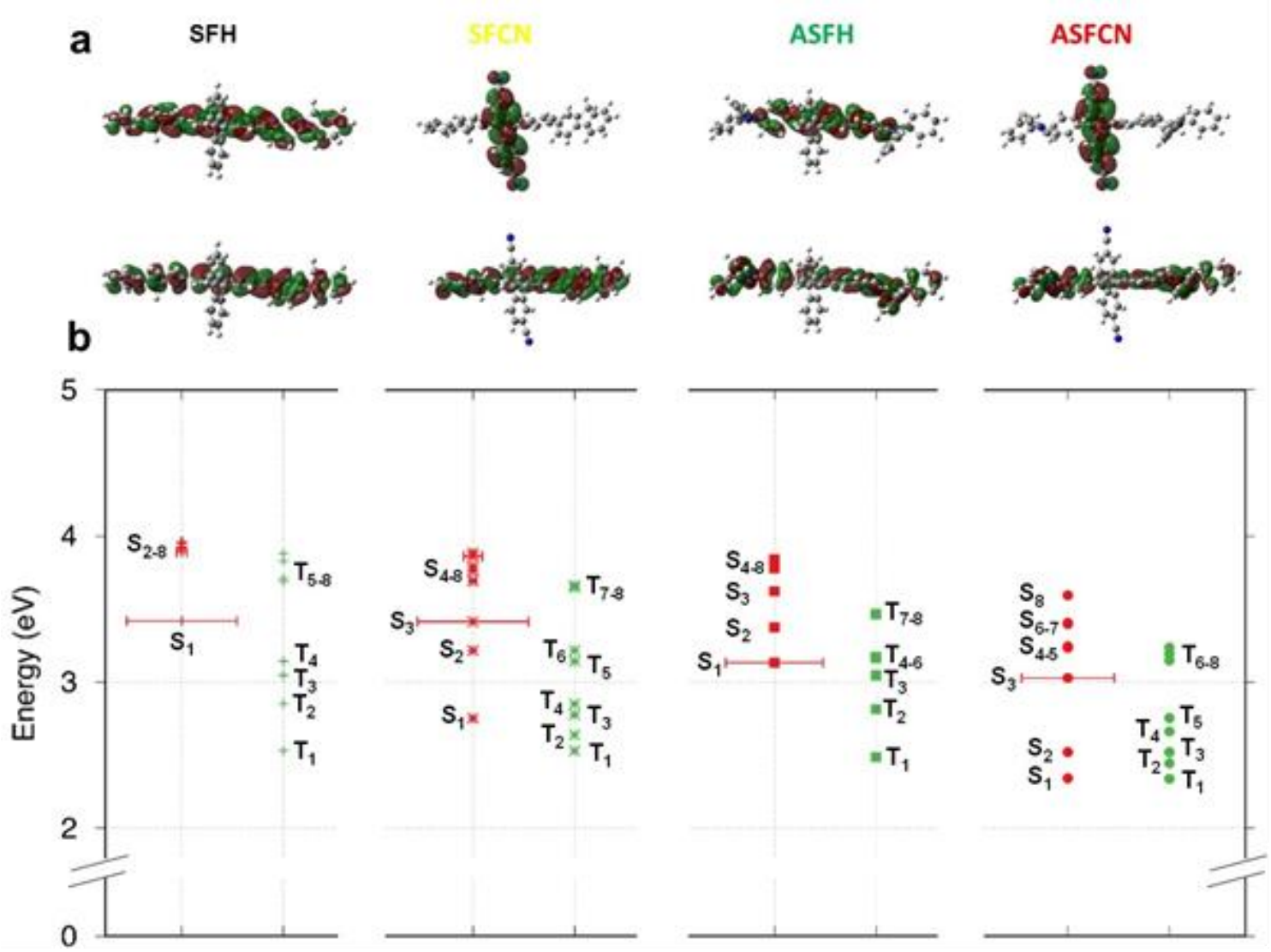

Figure 6. Electronic structure of model oligomers. (a) HOMO (bottom) and LUMO (top) of model trimers calculated using B3LYP/6-3119*. The D-oA motif results in complete localisation of the LUMO on the orthogonal fluorene unit. (b) Singlet and triplet states calculated by linear-response time-dependent density-functional-theory (LR-TD-DFT) at B3LYP/6-319*. The width of the state indicates the absorption oscillator strength, plotted logarithmically, indicating the large $\mathrm{Pi}-\mathrm{Pi}^{*}$ transition which will dominate optical absorption. The numbering is by order of energy, separately for both singlet and triplet states. 
The orthogonal acceptor introduces additional chargetransfer states below the main $\pi-\pi^{*}$ backbone transition. This backbone transition remains the strongest absorbing transition (by 100ox). Once absorbed there is an energetic driving force for the backbone $\pi^{*}$ exciton to transfer to the orthogonal acceptor, forming a lower energy CT state. These states are quasi-degenerate triplets and singlets. The orthogonal alignment of the moieties across the Spiro unit makes the frontier molecular orbitals (composed of Picharacter) orthogonal. This lack of molecular orbital overlap reduces the oscillator strength of this excited state, but also reduces the exchange energy that separates states of a Singlet and Triplet character. Though with TDDFT we cannot accurately predict the splitting in energy of the Singlet and Triplet states, the lack of splitting indicates that the overlap between the hole and electron within the excited state has tended towards zero, agreeing with our orbital analysis. In SFCN, these are states $\mathrm{S}_{1}$ and $\mathrm{T}_{2}$, slightly above the $3\left(\pi-\pi^{*}\right)$ of the backbone (T1). In ASFCN, these are states $\mathrm{S} 1$ and $\mathrm{T} 1$; the lowest-lying excited states of the material. In both cases, there is clearly a much richer set of excited states in the D-oA polymers to facilitate orbital mixing. Therefore, the weakly donating SFCN polymer retains the $3\left(\pi-\pi^{*}\right)$ as the lowest energy excited state meaning that the singlet-triplet energy gap is reduced to the energetic difference between the newly created ${ }^{1} \mathrm{CT}$ state and the previously existing $3\left(\pi-\pi^{*}\right)$. In the case of the stronger donating ASFCN the newly created singlet and triplet CT states are considerably below the $3\left(\pi-\pi^{*}\right)$, and due to the highly orthogonal nature of the spirocyclic charge transfer states we can assume $\Delta \mathrm{E}_{\text {ST }}$ to be very small $(<0.1 \mathrm{eV})$ as has been observed for small molecule spirocyclic TADF materials. ${ }^{32}$

\section{Discussion}

Our combined spectroscopic and computational results demonstrate the utility of our approach to reducing $\Delta \mathrm{E}_{\mathrm{ST}}$ in conjugated polymers. The presence of the spirobifluorene unit on its own does not perturb the photophysics of the conjugated polymer in any significant manner, as the HOMO and LUMO are both delocalised along the backbone. Incorporation of the strongly electron-withdrawing -CN groups creates new low-energy CT states which mix to a varying degree (dependent on 'push' from the main chain) with the main-chain ${ }^{1}\left(\pi-\pi^{*}\right)$ state. These CT states are composed of a delocalised ground state electron on the polymer backbone and an excited electron localised on the orthogonal acceptor. Consequently, the exchange energy is significantly reduced and we observe enhanced triplet formation. In the strongest push-pull D-oA material ASFCN, these effects combine to enable thermally activated RISC from the $3\left(\pi-\pi^{*}\right)$ state back into the weakly emissive CT state when triplet relaxation can be suppressed.

Our results also allow unprecedented insight into the underlying mechanism of triplet formation and RISC. In SFCN, in which the energy levels are relatively well separated, ${ }^{1}\left(\pi-\pi^{*}\right)$ convert predominantly into CT states, allowing for efficient formation of a lower-energy $3\left(\pi-\pi^{*}\right)$ states. Residual ${ }^{1}\left(\pi-\pi^{*}\right)$ states decay radiatively, breaking Kasha's rule as they are not the lowest excited singlet state. The rates and efficiencies of these processes are strongly affected by interchain interactions and restriction of the polymer conformation and vibrational degrees of freedom in the solid state, ${ }^{33}$ although the same basic pathway applies. Therefore SFCN is not capable of efficient RISC but instead leads to a novel route to generate polymer triplet excitons. In ASFCN, we observe signs that triplet formation and, in thin films, RISC, are mediated by excited-state mixing. The absorption red-shift and reduction in oscillator strength upon - $\mathrm{CN}$ substitution point to significant $\mathrm{CT}$ character in the initial ${ }^{1}\left(\pi-\pi^{*}\right)$ state, as in standard main-chain donoracceptor polymers. This state rapidly relaxes into the lower-energy CT state detected in photoluminescence. The observed correlation between CT and $3\left(\pi-\pi^{*}\right)$ transient absorption features suggests that this small energetic separation enables strong mixing between the two states. The character of this CT-3( $\left.\pi-\pi^{*}\right)$ mixture also evolves slightly over its lifetime, which we speculate could be attributed to relaxation of the low-energy vibrational modes coupling the two potential energy surfaces. ${ }^{34}$ At no point do we identify a pure, diabatic triplet or CT configuration, but these may be transiently populated, indicating the conventional picture of RISC may not be as simple as suggested. ${ }^{33}, 35-36$ It is this mixing with intermediate CT states which enables RISC to occur: the CT component of CT-3 $\left(\pi-\pi^{*}\right)$ enables a relatively fast (thermally activated) spin flip, resulting in a ${ }^{1} \mathrm{CT}$ configuration which in turn can emit via mixing with the ${ }^{1}\left(\pi-\pi^{*}\right)$ state. Our results are broadly in agreement with recent work on small molecule TADF systems which suggest that having a substantial CT contribution to $T_{1}$ and that the energetic proximity of a $\left(\pi-\pi^{*}\right) \mathrm{T}_{2}$ state enhances RISC. ${ }^{32}$ Given the success of donor-acceptor motifs to engineer efficient RISC in small molecules, we expect that the complex mechanism described here may be broadly applicable to other materials capable of TADF

\section{Conclusions}

We have demonstrated a concept which allows for the modification of standard conjugated polymers into ones with entirely new photophysics. This has been done by introducing orthogonal acceptor groups at the bridgehead position of alternating fluorene repeat units. This results in the creation of CT states below the main optically allowed $\pi-\pi^{*}$ transition, dramatically altering the excited state properties and reducing $\triangle \mathrm{E}_{\mathrm{ST}}$. In SFCN, these new CT states are higher in energy than the $3\left(\pi-\pi^{*}\right)$ and result in efficient triplet generation with ultra-long lifetimes, even at room temperature. Such materials could present an excellent platform to study the electronic properties of triplet excitons in more detail, or to harvest them for photochemistry such as singlet oxygen generation or photocatalysis. In the case of ASFCN, these new CT states are below the $3\left(\pi-\pi^{*}\right)$ and allow for TADF to occur via RISC, enabling a detailed transient absorption study of this little-understood phenomenon. Fused aromatic moieties with a bridgehead, as we have used here, are amongst the most common synthetic motif in conjugated polymers (eg. fluorene, cyclopentadithiophene, indacenodithiophene). Our D-oA motif can thus be easily applied to an entire library 
of structures, leading to a new class of materials with tuneable $\Delta \mathrm{E}_{\mathrm{ST}}$. A preliminary screen of materials using TD-DFT shows that using this approach it is possible to fully manipulate $\Delta \mathrm{E}_{\mathrm{ST}}$ across a wide energetic range and to vary the band-gap across the visible spectrum (Fig. $S_{7}$ ). There are two key advantages to the donor-orthogonal-acceptor design; firstly it allows for an additional dimension of tunability (energetically and morphologically) via conjugated polymer backbone manipulation and secondly, charge transport routes along the polymer backbone are maintained allowing chemists to enter a previously unexplored design space in conjugated polymer synthesis.

\section{ASSOCIATED CONTENT}

Synthesis and characterization of polymers, additional spectroscopic measurements and theoretical calculations. This material is available free of charge via the Internet at http://pubs.acs.org.

\section{AUTHOR INFORMATION}

\section{Corresponding Authors}

*a.musser@sheffield.ac.uk

*rhfio@cam.ac.uk

*h.bronstein@ucl.ac.uk

Present Addresses

† Dr Andrew Musser

The Department of Physics and Astronomy

Hicks Building

Hounsfield Road

Sheffield, $\mathrm{S}_{3} 7 \mathrm{RH}$

United Kingdom

Author Contributions

$\$$ These authors contributed equally.

\section{ACKNOWLEDGMENT}

Part of this work was funded by EU project 679789 CONTREX. AJM was supported by the EPSRC (EP/Mo1o83X). HLS was supported by the Winton Programme for the Physics of Sustainability.

\section{REFERENCES}

(1) Köhler, A.; Beljonne, D., Adv. Funct. Mater. 2004, 14, 11.

(2) Monkman, A. P.; Burrows, H. D.; Hartwell, L. J.; Horsburgh, L. E.; Hamblett, I.; Navaratnam, S., Phys. Rev. Lett. 2oo1, 86, 1358.

(3) Andernach, R.; Utzat, H.; Dimitrov, S. D.; McCulloch, I.; Heeney, M.; Durrant, J. R.; Bronstein, H., J. Am. Chem. Soc. 2015, $137,10383$.

(4) Rao, A.; Chow, P. C. Y.; Gelinas, S.; Schlenker, C. W.; Li, C.-Z.; Yip, H.-L.; Jen, A. K. Y.; Ginger, D. S.; Friend, R. H., Nature 2013, 500, 435 .

(5) Baldo, M. A.; O'Brien, D. F.; You, Y.; Shoustikov, A.; Sibley, S.; Thompson, M. E.; Forrest, S. R., Nature 1998, 395, 151.

(6) Kasemann, D.; Brückner, R.; Fröb, H.; Leo, K., Phys. Rev. $B$ 2011, 84, 115208 .

(7) Uoyama, H.; Goushi, K.; Shizu, K.; Nomura, H.; Adachi, C., Nature 2012, 492, 234.
(8) Wang, J.; Chepelianskii, A.; Gao, F.; Greenham, N. C., Nat. Commun. 2012, 3, 1191.

(9) Nikolaenko, A. E.; Cass, M.; Bourcet, F.; Mohamad, D.; Roberts, M., Adv. Mater. 2015, 27, 7236.

(10) Luo, J.; Xie, G.; Gong, S.; Chen, T.; Yang, C., Chem. Commun. 2016, 52, 2292.

(11) Lee, S. Y.; Yasuda, T.; Komiyama, H.; Lee, J.; Adachi, C., Adv. Mater. 2016, 28, 4019.

(12) Yu, W. L.; Pei, J.; Huang, W.; Heeger, A. J., Adv. Mater. 2000, 12,828 .

(13) Morris, J. V.; Mahaney, M. A.; Huber, J. R., J. Phys. Chem. 1976, 8o, 969 .

(14) Méhes, G.; Goushi, K.; Potscavage Jr, W. J.; Adachi, C., Org. Electron. 2014, 15, 2027.

(15) Méhes, G.; Nomura, H.; Zhang, Q.; Nakagawa, T.; Adachi, C., Angew. Chem. Int. Ed. 2012, 51, 11311.

(16) Png, R.-Q.; Chia, P.-J.; Tang, J.-C.; Liu, B.; Sivaramakrishnan, S.; Zhou, M.; Khong, S.-H.; Chan, H. S. O.; Burroughes, J. H.; Chua, L.-L.; Friend, R. H.; Ho, P. K. H., Nat. Mater. 2010, 9, 152.

(17) King, S. M.; Hintschich, S. I.; Dai, D.; Rothe, C.; Monkman, A. P., J. Phys. Chem. C 2007, 111, 18759.

(18) Aydemir, M.; Jankus, V.; Dias, F. B.; Monkman, A. P., J. Phys. Chem. C 2015, 119, 5855 .

(19) Hintschich, S. I.; Rothe, C.; King, S. M.; Clark, S. J.; Monkman, A. P., J. Phys. Chem. B 2008, 112, 16300.

(20) dos Santos, P. L.; Dias, F. B.; Monkman, A. P., J. Phys. Chem. C 2016, 120, 18259.

(21) Wang, X.; Zhao, L.; Shao, S.; Ding, J.; Wang, L.; Jing, X.; Wang, F., Polym. Chem. 2014, 5, 6444.

(22) Wan, Y.; Guo, Z.; Zhu, T.; Yan, S.; Johnson, J.; Huang, L., Nat. Chem. 2015, 7, 785 .

(23) Devižis, A.; Serbenta, A.; Meerholz, K.; Hertel, D.; Gulbinas, V., J. Chem. Phys. 2009, 131, 104902.

(24) Rothe, C.; King, S. M.; Monkman, A. P., Phys. Rev. B 2005, 72, 085220 .

(25) Stern, H. L.; Musser, A. J.; Gelinas, S.; Parkinson, P.; Herz, L. M.; Bruzek, M. J.; Anthony, J.; Friend, R. H.; Walker, B. J., Proc. Natl. Acad. Sci. 2015, 112, 7656.

(26) Korovina, N. V.; Das, S.; Nett, Z.; Feng, X.; Joy, J.; Haiges, R.; Krylov, A. I.; Bradforth, S. E.; Thompson, M. E., J. Am. Chem. Soc. 2016, 138, 617.

(27) Pensack, R. D.; Ostroumov, E. E.; Tilley, A. J.; Mazza, S.; Grieco, C.; Thorley, K. J.; Asbury, J. B.; Seferos, D. S.; Anthony, J. E.; Scholes, G. D., J. Phys. Chem. Lett. 2016, 7, 2370.

(28) Bakulin, A. A.; Morgan, S. E.; Kehoe, T. B.; Wilson, M. W. B.; Chin, A. W.; Zigmantas, D.; Egorova, D.; Rao, A., Nat. Chem. 2016, 8, 16.

(29) Saragi, T. P. I.; Spehr, T.; Siebert, A.; Fuhrmann-Lieker, T.; Salbeck, J., Chem. Rev. 2007, 107, 1011.

(30) Hait, D.; Zhu, T.; McMahon, D. P.; Van Voorhis, T., J. Chem. Theory Comput. 2o16, 12, 3353.

(31) Petersilka, M.; Gross, E. K. U.; Burke, K., Int. J. Quantum Chem 2000, 80, 534 .

(32) Samanta, P. K.; Kim, D.; Coropceanu, V.; Brédas, J.-L., J. Am. Chem. Soc. 2017, 139, 4042.

(33) Gibson, J.; Monkman, A. P.; Penfold, T. J., ChemPhysChem 2016, 17, 2956.

(34) Etherington, M. K.; Gibson, J.; Higginbotham, H. F.; Penfold, T. J.; Monkman, A. P., Nat. Commun. 2016, 7, 13680.

(35) Zhang, Q.; Li, B.; Huang, S.; Nomura, H.; Tanaka, H.; Adachi, C., Nat. Photon. 2014, 8, 326.

(36) Jankus, V.; Aydemir, M.; Dias, F. B.; Monkman, A. P., Adv. Sci. 2016, 3, 1500221. 
Insert Table of Contents artwork here
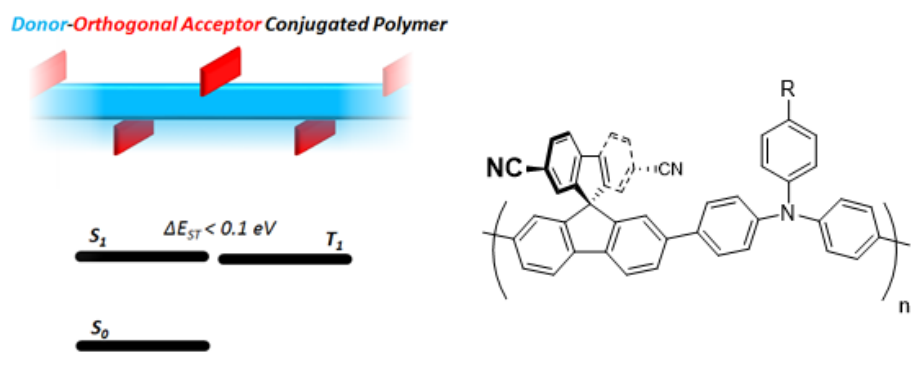